\title{
㡘磷锰铁矿中配位基氞离子的 无序缺席效应
}

\author{
李 哲 张如柏* 赫 伟 于 莉 \\ (中国科学院地质研究所, 北京)
}

氧磷锰铁矿 $(\mathrm{Mn}, \mathrm{Fe}, \mathrm{Mg}, \mathrm{Ca})_{2} \mathrm{FPO}$ 是一种不常见的磷酸盐矿物, 常常出现在伟昆岩 中. 对它的矿物学和结构以及穆斯堡尔谱已经做过研究 ${ }^{[1-3]}$. Kostiner 等 ${ }^{[3]}$ 的研究表明, 它的 室温穆斯堡尔谱由两个 $\mathrm{Fe}^{2+}$ 四极双峰组成, 其线宽分别为 $0.35 \mathrm{~mm} / \mathrm{s}$ 和 $0.72 \mathrm{~mm} / \mathrm{s}$, 后者相当 宽. 本文对偂磷钙铁矿进行了室温和液氮温度下的穆斯堡尔谱学研究, 首次发现由于偂离子 的无序缺席而形成的谱线分裂现象, 作者建议把这一现象称为配位基阴离子无序缺席效应.除 了电子非局域化和次近邻效应之外, 这是一种新的可以使用穆斯堡尔效应来研究的矿物中的 精细相互作用.

\section{一、实验方法}

本文所研究的偂磷镋铁矿 $\left(\mathrm{Mn}_{1.52} \mathrm{Fe}_{0.162}^{2+} \mathrm{Mg}_{0.02} \mathrm{Ca}_{0.08}\right)_{1.78}\left(\mathrm{P}_{0.88} \mathrm{Si}_{0.02}\right)_{0.89} \mathrm{O}_{4}\left(\mathrm{~F}_{0.76}(\mathrm{OH})_{0.16}\right)_{0.92}$ 产 自我国山西 ${ }^{[1]}$. 使用湿式化学分析方法对矿物进行化学分析, 并且使用 $\mathrm{X}$-射线衍射对其纯度 进行了检测。

本研究使用 MS-2 型穆斯堡尔谱仪记录镜像谱,谱仪与 1024 多道分析仪联用. 约 $10 \mathrm{mC}_{i}$ 的 ${ }^{57} \mathrm{Co}(\mathrm{Rh})$ 做为放射源, $\mathrm{NaI}(\mathrm{Tl})$ 内淿计数器做为探测器. 使用最小二乘法计算机程序对 所测谱拟合, 在拟合过程中限制 $\mathrm{Fe}^{2+}$ 双峰宽度相等.

\section{二、结果和讨论}

图 1 示出了该氟磷镇铁矿在 $298 \mathrm{~K}$ 和 $77 \mathrm{~K}$ 下的穆斯堡尔谱, 它们都是由 $\mathrm{A}, \mathrm{B}, \mathrm{C}, \mathrm{D}$ 和 $\mathrm{E}$ 五个双峰组成. 表 1 列出了该氟磷镇铁矿在 $298 \mathrm{~K}$ 和 $77 \mathrm{~K}$ 下的穆斯堡尔参数. 由表 1 所列参 数可以看出, 双峰 $\mathrm{A}, \mathrm{B}, \mathrm{C}$ 和 $\mathrm{D}$ 是由 $\mathrm{Fe}^{2+}$ 离子形成的, 而双峰 $\mathrm{E}$ 则归结为少量的 $\mathrm{Fe}^{3+}$ 离子. 这样, 该氟磷镇铁矿的结晶化学式应修正为 $\left(\mathrm{Mn}_{1.52} \mathrm{Fe}_{0.122}^{2+} \mathrm{Fe}_{0.010}^{3+} \mathrm{Mg}_{0.02} \mathrm{Ca}_{0.08}\right)_{1.78}\left(\mathrm{P}_{0.88} \mathrm{Si}_{0.02}\right)_{0.89} \mathrm{O}$, $\left(\mathrm{F}_{0.76}(\mathrm{OH})_{0.16}\right)_{0.92 \text {. }}$.

对氟磷镇铁矿的结构研究表明, 它的空间群为 $12 / a$, 属单斜晶系, $Z=4^{[2]}$. 四个O配 位于 $\mathrm{P}$ 离子, 构成 $\mathrm{P}$ 四面体, 其 $\mathrm{P}-\mathrm{O}$ 平均间距为 $1.536 \AA$. 阳离子占据两种结晶学上不等效 位置 $\mathrm{M} 1$ 和 $\mathrm{M} 2$, 它们均为四个 $\mathrm{O}$ 和四个半填充的 $F$ 位置所配位的八面体, 其中三个 $F$ 离子与 阳离子之间的间距为正常值, 另一个间距较大, 把这四个半填充的 $F$ 位置表示为 $F(1), F(2)$, $F(1)^{\prime}$ 和 $F(2)^{\prime}$.

氧磷镇铁矿 $M 2$ 八面体共边形成平行于 $a$ 轴的 $Z \mathrm{igzag}$ 链,这些链与 M1 八西体共边和环

本文 1986 年 8 月 28 日收到.

* 成都地质学院. 


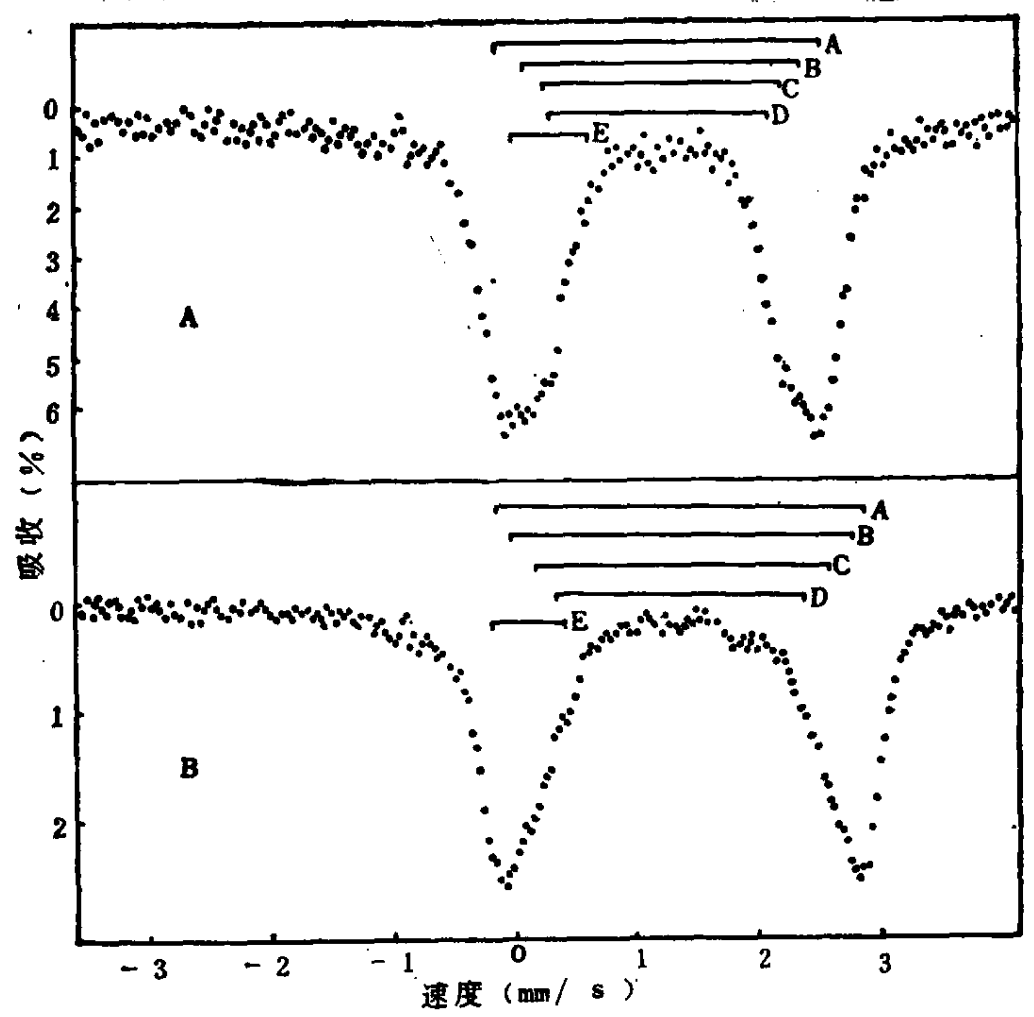

图 1 氧磷钴铁矿在 $298 \mathrm{~K}$ (A) 和 $77 \mathrm{~K}$ (B) 下的穆斯堡尔徣

表 1 氛磷锰铁矿的穆斯堡尔参数

\begin{tabular}{|c|c|c|c|c|c|c|}
\hline 温度 & I.S. $(\mathrm{mm} / \mathrm{s})$ & Q.S. $(\mathrm{mm} / \mathrm{s})$ & $\Gamma(\mathrm{mm} / \mathrm{s})$ & 面积\% & 指派 & $x^{2}$ \\
\hline \multirow{5}{*}{$298 \mathrm{~K}$} & 1.20 & 2.68 & \multirow{4}{*}{0.37} & 40 & $\left\{\begin{array}{l}\operatorname{M1}\left(F(2), F(1)^{\prime}\right) \\
\operatorname{M} 2\left(F(2), F(1)^{\prime}\right.\end{array}\right.$ & \multirow{5}{*}{1.33} \\
\hline & 1.22 & 2.31 & & 22 & $\left\{\begin{array}{l}\operatorname{M1}\left(F(1), F(1)^{\prime}\right) \\
\operatorname{M} 2\left(F(2), F(2)^{\prime}\right.\end{array}\right.$ & \\
\hline & 1.22 & 1.98 & & 19 & $\left\{\begin{array}{l}\operatorname{M1}\left(F(1), F(2)^{\prime}\right) \\
\operatorname{M} 2\left(F(1), F(1)^{\prime}\right)\end{array}\right.$ & \\
\hline & 1.22 & 1.78 & & 14 & $\left\{\begin{array}{l}\operatorname{M1}\left(F(2), F(2)^{\prime}\right) \\
\operatorname{M}^{\prime}\left(F(1), F(2)^{\prime}\right)\end{array}\right.$ & \\
\hline & 0.42 & 0.31 & 0.80 & 5 & $\mathrm{M} 1, \mathrm{M} 2$ & \\
\hline \multirow{5}{*}{$77 \mathrm{~K}$} & 1.31 & 3.05 & \multirow{4}{*}{0.35} & 38 & $\left\{\begin{array}{l}\operatorname{M1}\left(F(2), F(1)^{\prime}\right) \\
M_{2}\left(F(2), F(1)^{\prime}\right)\end{array}\right.$ & \multirow{5}{*}{1.40} \\
\hline & 1.33 & 2.78 & & 26 & $\left\{\begin{array}{l}\mathrm{M1}\left(F(1) F(1)^{\prime}\right) \\
\mathrm{M}_{2}\left(F(2), F(2)^{\prime}\right)\end{array}\right.$ & \\
\hline & 1.33 & 2.41 & & 19 & $\left\{\begin{array}{l}\operatorname{M1}\left(F(1), F(2)^{\prime}\right) \\
\operatorname{M} 2\left(F(1), F(1)^{\prime}\right)\end{array}\right.$ & \\
\hline & 1.31 & 2.05 & & 9 & $\begin{array}{l}\mathrm{M} 1\left(F(2), F(2)^{\prime}\right) \\
\mathrm{M} 2\left(F(1), F(2)^{\prime}\right)\end{array}$ & \\
\hline & 0.46 & 0.62 & 0.80 & 7 & $\mathrm{M} 1, \mathrm{M} 2$ & \\
\hline
\end{tabular}

立 $P$ 四面体共顶连接. 同时, $M 1$ 八面体共边形成平行于 $b$ 轴的链. 图 2 示出了沿 $b$ 轴投影 的结构.

一般假定一种结构位置上的 $\mathrm{Fe}^{2+}$ 或 $\mathrm{Fe}^{3+}$ 离子会在矿物的穆斯堡尔谱上形成一个双峰. 然 而, 使用二个双峰拟合室温穆斯堡尔谱所给出的线宽为 $0.41 \mathrm{~mm} / \mathrm{s}$, 而且 $\chi^{2}=1.51$ 也超出统 计上可以接受的值. 当使用四个 $\mathrm{Fe}^{2+}$ 双峰和一个 $\mathrm{Fe}^{3+}$ 双峰拟合该谱时, 线宽减至 $0.37 \mathrm{~mm} / \mathrm{s}$, $x^{3}$ 值也减至统计上可以接受值. 由此可以看出, 必须使用一种新的模型来解释䀜磷镇铁矿穆 


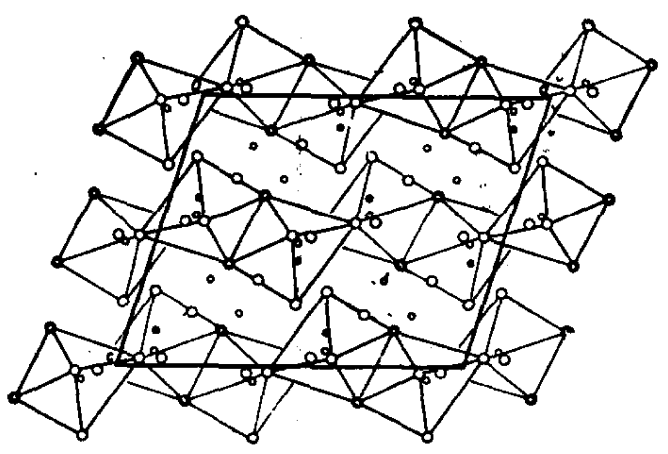

图 2 磷锰铁矿沿 6 轴的结构投影 （实心圆表示 $P$ 要子，大圆代表草离子，双圆代表 F离子，小圆代表阳离子(据文献[2])

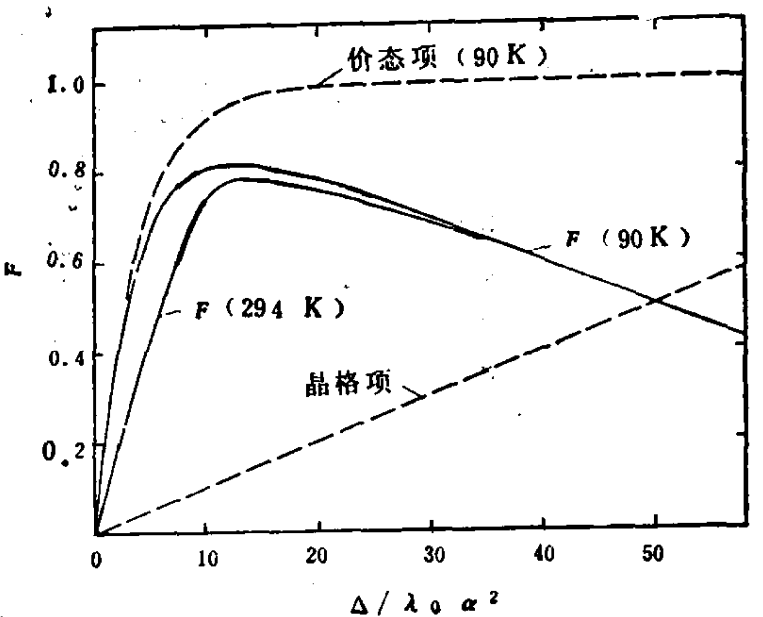

图 3 缩减函数 $F$ 与八面体畸变程 度 $\Delta / \lambda_{0} \alpha^{2}$ 之间的关系(琚文献[5])

斯堡尔谱中所观测到的多重四极双峰.

已经在钻辉石和绿辉石等矿物中观测到次近邻效应所形成的四极双峰 ${ }^{[4]}$. Ingallas ${ }^{[9]}$ 已 经确定了如图 3 所示的四极分裂缩减函数 $F$ 与八面体畸变程度之间的关系.由图 3 可以看出， 缩减函数 $F$ 在开始时随畸变程度增大很快. 然而, 在缩减函数 $F$ 达到极值后, 随畸变程度增 大而缓慢下降. 辉石中 M1 八面体的䗁变程度小，它与缩减函数 $F$ 之间关系落在图 3 所示的 黑线部分, 这部分曲线斜率为正而且值较大, 畸变程度的微小变化会引起缩减函数 $F$ 的很大 变化. 这样, 容易观测到由次近邻作用而形成的多重双峰. 表 2 列出了矿物的平方伸长度 $\Delta$ 和角度变化 $\sigma^{2}$, 它们都是用来表示八面体畸变程度的. 可以看出, 辉石 $\mathrm{M} 1$ 八面体的 $\Delta$ 和 $\sigma^{2}$ 值较小,而辉石 M2 多面体相应的值较大, 它落在图 3 曲线斜率为负的平缓区域. 因此, 在 钙

表 2 矿物中八面体的平方伸长度和角度变化

\begin{tabular}{|c|c|c|c|c|}
\hline 矿物 & 位 置 & $\Delta^{*}$ & $\sigma^{2 * *}$ & 文献 \\
\hline 正铁辉石 & $\begin{array}{l}\text { M1 } \\
\text { M2 }\end{array}$ & $\begin{array}{l}0.0004 \\
0.008 t\end{array}$ & $\begin{array}{r}25.65 \\
167.12\end{array}$ & {$[6]$} \\
\hline 周磷铁矿 & $\begin{array}{l}\operatorname{M1}\left(F(1), F(1)^{\prime}\right) \\
\operatorname{M1}\left(F(1), F(2)^{\prime}\right) \\
\operatorname{M1}\left(F(2), F(1)^{\prime}\right) \\
\operatorname{M1}\left(F(2), F(2)^{\prime}\right) \\
\operatorname{M} 2\left(F(1), F(1)^{\prime}\right) \\
\operatorname{M} 2\left(F(1), F(2)^{\prime}\right) \\
\operatorname{M} 2\left(F(2), F(1)^{\prime}\right) \\
\operatorname{M} 2\left(F(2), F(2)^{\prime}\right)\end{array}$ & $\begin{array}{l}0.0125 \\
0.0014 \\
0.0139 \\
0.0018 \\
0.0016 \\
0.0012 \\
0.0125 \\
0.0122\end{array}$ & $\begin{array}{l}273.79 \\
324.19 \\
291.99 \\
289.10 \\
207.28 \\
124.54 \\
228.58 \\
164.02\end{array}$ & [2] \\
\hline
\end{tabular}

* $\Delta=\frac{1}{6} \sum_{i=1}^{\circ}\left(\frac{l_{1}-l_{m}}{l_{m}}\right)^{2}, l_{i}$ 为 M-O 间距, $l_{m}$ 为平均间距.

** $\sigma^{2}=\sum_{i=1}^{12}\left(\theta_{i}-90\right)^{2} / 11, \theta_{i}$ 为八面体键角.

辉石和钙铁辉石-铁辉石中, 没有观测到 $\mathrm{M} 2$ 位置上 $\mathrm{Fe}^{2+}$ 的次近邻效应, 而观测到 $\mathrm{M} 1$ 位置 $\mathrm{Fe}^{2+}$ 的次近邻效应. 
对氟磷镇铁矿来说, $\mathrm{Fe}^{2+}$ 离子占据结晶学上不等效的 $\mathrm{M} 1$ 和 $\mathrm{M} 2$ 位置. 然而, 由于 $\mathrm{M} 1$ 和 M2 位置分别有四个半填充的 $F$ 离子与之配位, 因此, $F$ 离子的无序缺席将会形成如表 2 所示 的八种亚晶位. 表 2 列出了 $M 1$ 和 M2 各种亚晶位的 $\Delta$ 和 $\sigma^{2}$ 值. 可以看出, 与辉石 $M 1$ 的值 相比, 这些值都很大, 因此, 预计它将落在 $F$ 曲线 (图 3)斜率为负的区域. 由于这一区域中 $F$ 对畸变程度并不灵敏,因此将观测不到次近邻效应.

对处在 $\mathrm{M} 1$ 和 $\mathrm{M} 2$ 位置上 $\mathrm{Fe}^{2+}$ 离子来说, $F$ 为近邻离子, 它对 $\mathrm{Fe}^{2+}$ 离子处电场梯度的贡 献比次近邻大, 因此, $F$ 离子的无序缺席将会进一步形成亚晶位, 并且有可能使穆斯堡尔谱线 分裂而形成相应于各种亚晶位的多重四极双峰, 这就是本文作者提出的 $F$ 离子无序缺席效应. 根据表 2 所列出的氟磷镇铁矿中 M1 和 M2 亚晶位的 $\Delta$ 和 $\sigma^{2}$ 值, 试探性地给出表 1 的指派。由 于某些亚晶位的 $\sigma^{2}$ 值相似, 因此只分出四个由于 $F$ 离子无序缺席而形成的双峰. 由表 1 还可 看出, $298 \mathrm{~K}$ 和 $77 \mathrm{~K}$ 的穆斯堡尔结果符合得很好.

$F$ 离子无序缺席效应是一种新的可以使用穆斯堡尔效应研究的精细作用．在实际上，任 何一种配位基阳离子的无序缺席都会形成畸变程度不同的亚晶位, 因而有可能在穆斯堡尔说 中形成多重四极双峰.

致谢：作者感谢叶大年教授的热情鼓励和有益的讨论.

[ 1 ] 张如柏、谢维基, 矿物岩石, 4(1984)，1：89--90.

[ 2] Waldrop, L., Z. Kristallogr., 130(1969), 1-4.

[3] Kostiner, E., Amer. Mineral., 57(1972), 1109-1114.

[ 4 ] Bancroft, G. M., J. Phys., Colloq. C2, 40(1979), 464-471.

[5] Ingallas,. R., Phys. Rev., 133A(1964), 787-795.

[6] Sueno, S., Cameron, M. and Prewite, C. T. Amer. Mineral., 61(1970), 38-53. 